Bernhard Strauß · Jochen Eckert Volker Tschuschke (Hrsg.)

Methoden der empirischen Gruppentherapieforschung 
Bernhard Strauß · Jochen Eckert

Volker Tschuschke (Hrsg.)

\section{Methoden der empirischen Gruppentherapieforschung}

Ein Handbuch

Springer Fachmedien Wiesbaden GmbH 
Die Deutsche Bibliothek - CIP-Einheitsaufnahme

Methoden der empirischen Gruppentherapieforschung:

ein Handbuch /

Bernhard Strauss ... (Hrsg.).

ISBN 978-3-531-12695-1

ISBN 978-3-663-10786-6 (eBook)

DOI 10.1007/978-3-663-10786-6

NE: Strauss, Bernhard (Hrsg.)

Alle Rechte vorbehalten

(C) 1996 Springer Fachmedien Wiesbaden

Ursprünglich erschienen bei Westdeutscher Verlag GmbH, Opladen 1996

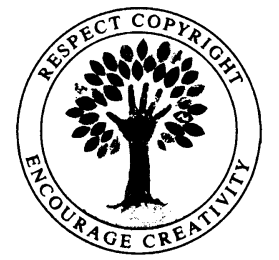

Das Werk einschließlich aller seiner Teile ist urheberrechtlich geschützt. Jede Verwertung außerhalb der engen Grenzen des Urheberrechtsgesetzes ist ohne Zustimmung des Verlags unzulässig und strafbar. Das gilt insbesondere für Vervielfältigungen, Übersetzungen, Mikroverfilmungen und die Einspeicherung und Verarbeitung in elektronischen Systemen.

Umschlaggestaltung: Horst Dieter Bürkle, Darmstadt

Titelbild: Paul Klee, Maibild abstract, 1925, 120 (C 0), 41,5 x 49,5 cm; Öl auf Karton; Metropolitan Museum of Art, New York, NY, The Berggruen Klee Collection, Inv. Nr. 1984.315.42.; @ VG Bild-Kunst, Bonn 1996

Gedruckt auf säurefreiem Papier

ISBN 978-3-531-12695-1 
Dieses Buch ist dem Andenken an

Susanne Davies-Osterkamp (1943-1995)

gewidmet. 


\section{Inhaltsverzeichnis}

1 Einführung: Die Gruppe - Ein Stiefkind der Psychotherapieforschung?

Bernhard Strau $\beta$, Jochen Eckert, Volker Tschuschke

Grundlagen:

Forschungsstrategien in der Gruppenpsychotherapie

2 Indikation und Prognose

Jochen Eckert

3 Ergebnisforschung in der Gruppenpsychotherapie

Bernhard Strau $\beta$

4 Prozeß-Ergebnis-Zusammenhänge und Wirkfaktorenforschung

Volker Tschuschke

Spezifische Forschungsmethoden:

Statusdiagnostik interpersonaler Prognosefaktoren

5 Das Inventar zur Erfassung interpersonaler Probleme (IIP)

Bernhard Strauß, Hans Kordy

6 Psychological Mindedness Assessment Procedure (PMAP)

Mary MacCallum, William E. Piper

Wirkfaktorendiagnostik

7 Das Gruppenwirkfaktoren-Q-Sort nach Yalom

Jochen Eckert

8 Der Düsseldorfer Wirkfaktorenfragebogen - ein Instrument zur differentiellen Beschreibung von Gruppenpsychotherapien Susanne Davies-Osterkamp

9 Die Kieler Gruppenpsychotherapie-Prozeß-Skala (KGPPS) zur Fremdbeurteilung therapeutischer Faktoren in der Gruppenpsychotherapie Bernhard Strauß, Manuela Burgmeier-Lohse, Regina Rohweder, Heike Wienands 
10 Zwei Verfahren zur Einschätzung der Wirksamkeit von

Gruppenpsychotherapie: Die Behandlungseinschätzung nach Froese und die Bewertungsskala nach Böttcher und Ott

Helga Hess

Prozeßdiagnostik

11 Gruppenerfahrungsbogen (GEB)

Jochen Eckert

12 Der Gruppenklima-Fragebogen (Group Climate Questionnaire, GCQ-S)

K. Roy MacKenzie

13 Potenz-Valenz-Polaritätenprofil nach Feldes

Michael Geyer, Günther Plöttner

14 Der Stuttgarter Bogen (SB)

Volker Tschuschke

15 Das Soziogramm nach Höck und Hess eine Methode zur Erfassung soziometrischer Strukturen im Gruppenprozeß Helga Hess

16 Die Hill-Interaktionsmatrix

Czeslaw Czabala, Celina Brykcynska

17 Das SYMLOG-Rating-Verfahren:

Interaktionsdiagostik im SYMLOG-Raum

Friedebert Kröger, Dieter Wälte, Arno Drinkmann

18 Das „ORLIK-Gitter“ zur Erfassung subjektiver Bewertungsstrukturen und ihrer Änderung im Gruppenpsychotherapieprozeß Andreas Lange, Volker Bracke

19 Die Anwendung der Grid-Technik in der Gruppentherapie-Prozeßdiagnostik Ana Catina, Beatrice Krämer

20 Die „Structural Analysis of Social Behavior“ (SASB) als

Methode zur Analyse von Gruppenprozessen

Manuela Burgmeier-Lohse

21 Das Zeichensystem zur Erfassung von Handlungsorientierung und Lageorientierung in verbalen Äußerungen (Z-HOLO) von Hartung Michael Bartschat, Mathias Fauth, Bernhard Strauß 
22 Der Gruppenprozeß unter der Lupe: Das Matrix-Representation-Grid Göran Ahlin

23 Anwendung der Methode des ,Zentralen Beziehungskonfliktthemas“(ZBKT) in der Gruppentherapie

Hermann Staats, Joachim Biskup .360

24 Gottschalk-Gleser-Sprachinhaltsanalyse Volker Tschuschke

25 Übersicht: Weitere Methoden der Gruppenpsychotherapieforschung Bernhard Strauß, Jochen Eckert, Volker Tschuschke

Sachverzeichnis. .399

Anschriften der Autoren .404 